\title{
On the Evaluation of Double Integrals
}

\author{
By Moshe Levin
}

\begin{abstract}
A cubature formula consisting of line integrals which is optimal on a set functions satisfying given boundary conditions is obtained. The line integrals of this formula may be evaluated by optimal quadrature formulas. The advantage of this formula over the optimal cubature formula with a rectangular lattice of knots is shown. This approach to optimal cubatures was stimulated by the idea of blending [1], [2].
\end{abstract}

1. Notations and Definitions. Let $L_{1 i} f(\cdot)=0, i=1, \ldots, r_{1}$, and $L_{2 j} f(\cdot)=0$, $j=1, \ldots, r_{2}$, be linear homogeneous boundary conditions for the interval $[0,1]$ such that the problems

$$
\left\{\begin{array} { l } 
{ y ^ { ( r ) } ( x ) = 0 , } \\
{ L _ { 1 i } y ( \cdot ) = 0 , \quad i = 1 , \ldots , r _ { 1 } , }
\end{array} \text { and } \left\{\begin{array}{l}
y^{(r)}(x)=0, \\
L_{2 j} y(\cdot)=0, \quad j=1, \ldots, r_{2},
\end{array}\right.\right.
$$

both have the unique solution $y(x) \equiv 0$, and let $g_{1}(x, t)$ and $g_{2}(x, t)$ be the Green's functions corresponding to problems (1) [4].

$$
\begin{aligned}
& \text { Let } \mathscr{D}=[0,1] \times[0,1], 1<p<\infty, p^{-1}+q^{-1}=1, \\
& \|f(\cdot)\|_{p}=\left(\int_{0}^{1}|f(x)|^{p} d x\right)^{1 / p}, \quad\|\varphi(\cdot, \cdot)\|_{p}=\left(\int_{0}^{1} \int_{0}^{1}|\varphi(x, y)|^{p} d x d y\right)^{1 / p} .
\end{aligned}
$$

We consider the following sets of functions:

$$
\begin{gathered}
W_{g_{k}}^{r} L_{p}=\left\{f(x): f^{(r)}(x) \text { piecewise continuous on }[0,1],\right. \\
\left.\left\|f^{(r)}(\cdot)\right\|_{p} \leqslant 1, L_{k i} f(\cdot)=0, i=1, \ldots, r_{k}\right\}, \quad k=1,2, \\
W_{g_{1} g_{2} L_{p}}^{2 r} L_{p}\left\{f(x, y): \frac{\partial^{l+s} f(x, y)}{\partial x^{l} \partial y^{s}}(l, s \leqslant r) \text { piecewise continuous on } \mathscr{D},\right. \\
\left\|\frac{\partial^{2 r} f(\cdot, \cdot)}{\partial x^{r} \partial y^{r}}\right\|_{p} \leqslant M, L_{1 i} f(\cdot, y) \equiv 0, i=1, \ldots, r_{1} ; \\
\left.L_{2 j} f(x, \cdot) \equiv 0, j=1, \ldots, r_{2}\right\} .
\end{gathered}
$$

The quadrature formula

$$
\int_{0}^{1} f(x) d x=\sum_{k=1}^{m} A_{k} f\left(x_{k}\right)+r(f), \quad 0 \leqslant x_{1}<\cdots<x_{m} \leqslant 1
$$

Received June 10, 1981; revised October 27, 1981.

1980 Mathematics Subject Classification. Primary 65D30; Secondary 65D32. 
is called optimal for the set $H$ of functions $f(x)$ if the coefficients $A_{k}$ and knots $x_{k}$ of the formula are chosen so that the quantity

$$
\sup _{f \in H}|r(f)|
$$

is minimal.

Designate

$$
\begin{aligned}
& A_{p 1}^{(m)}, \ldots, A_{p m}^{(m)} ; x_{p 1}^{(m)}, \ldots, x_{p m}^{(m)} ; r_{p 1}^{(m)} \\
& B_{p 1}^{(m)}, \ldots, B_{p m}^{(m)} ; y_{p 1}^{(m)}, \ldots, y_{p m}^{(m)} ; r_{p 2}^{(m)}
\end{aligned}
$$

the coefficients, knots, and the value (3) of the optimal formula (2) for the sets $W_{g_{1}}^{r} L_{p}$ and $W_{g_{2}}^{r} L_{p}$, respectively. It is known [4] that $r_{p 1}^{(m)}=O\left(m^{-r}\right), r_{p 2}^{(m)}=O\left(m^{-r}\right)$.

The formula

$$
\begin{aligned}
& \int_{0}^{1} \int_{0}^{1} f(x, y) d x d y=\sum_{k=1}^{m} \sum_{l=1}^{m} C_{k l} f\left(x_{k}, y_{l}\right)+R(f), \\
& \quad 0 \leqslant x_{1}<\cdots<x_{m} \leqslant 1,0 \leqslant y_{1}<\cdots<y_{m} \leqslant 1,
\end{aligned}
$$

is called the optimal formula for the set $F$ of functions $f(x, y)$ if its coefficients and knots are chosen so that the quantity

$$
R=\sup _{f \in F}|R(f)|
$$

has the least value.

It is shown in [3], [4] that the optimal formula (4) on the set $W_{g_{1} g_{2}}^{2 r} L_{2}$ has the coefficients $C_{k l}=A_{2 k}^{(m)} B_{2 l}^{(m)}$, the knots $x_{k}=x_{2 k}^{(m)}, y_{l}=y_{2 l}^{(m)}$, and the remainder $R=O\left(m^{-r}\right)$.

For the case $p \neq 2$ the optimal formula (4) on the set $W_{g_{1} g_{2}}^{2 r} L_{p}$ has not yet been found.

2. The Optimal Cubature Formula. We will find an optimal formula of the form

$$
\begin{aligned}
\int_{0}^{1} \int_{0}^{1} f(x, y) d x d y= & \sum_{k=1}^{n} \alpha_{k} \int_{0}^{1} f\left(x_{k}, y\right) d y+\sum_{j=1}^{n} \beta_{j} \int_{0}^{1} f\left(x, y_{j}\right) d x \\
& +\sum_{k=1}^{n} \sum_{j=1}^{n} \gamma_{k j} f\left(x_{k}, y_{j}\right)+E(f) \\
& 0 \leqslant x_{1}<\cdots<x_{n} \leqslant 1,0 \leqslant y_{1}<\cdots<y_{n} \leqslant 1
\end{aligned}
$$

for the set $W_{g_{1} g_{2}}^{2 r} L_{p}$. In other words, we will find the formula (5) with the least value of

$$
E=\sup _{f \in W_{8182}^{2 r} L_{p}}|E(f)| .
$$

THEOREM. The coefficients and knots

$$
\begin{aligned}
& \alpha_{k}=A_{p k}^{(n)}, \quad \beta_{j}=B_{p j}^{(n)}, \quad \gamma_{k j}=-A_{p k}^{(n)} B_{p j}^{(n)}, \\
& x_{k}=x_{p k}^{(n)}, \quad y_{j}=y_{p j}^{(n)}, \quad k, j=1, \ldots, n,
\end{aligned}
$$

and the estimate

$$
E=M r_{p 1}^{(n)} r_{p 2}^{(n)}
$$

are the coefficients, knots, and estimate of the optimal formula (5) on the set $W_{g_{1} g_{2}}^{2 r} L_{p}$. 
Proof. Let $f(x, y) \in W_{g_{1} g_{2}}^{2 r} L_{p}$. Then [4]

$$
f(x, y)=\int_{0}^{1} \int_{0}^{1} \frac{\partial^{2 r} f(t, u)}{\partial x^{r} \partial y^{r}} g_{1}(x, t) g_{2}(y, u) d t d u \text {. }
$$

Hence by (5)

$$
E(f)=\int_{0}^{1} \int_{0}^{1} \frac{\partial^{2 r} f(t, u)}{\partial x^{r} \partial y^{r}} K(t, u) d t d u
$$

where

$$
\begin{gathered}
K(t, u)=\varphi_{1}(t) \varphi_{2}(u)-\varphi_{2}(u) \sum_{k=1}^{n} \alpha_{k} g_{1}\left(x_{k}, t\right)-\varphi_{1}(t) \sum_{j=1}^{n} \beta_{j} g_{2}\left(y_{j}, u\right) \\
-\sum_{k=1}^{n} \sum_{j=1}^{n} \gamma_{k j} g_{1}\left(x_{k}, t\right) g_{2}\left(y_{j}, u\right), \\
\varphi_{l}(v)=\int_{0}^{1} g_{l}(x, v) d x, \quad l=1,2 .
\end{gathered}
$$

Using Hölder's inequality, we obtain from (8) that

$$
|E(f)| \leqslant M\|K(\cdot, \cdot)\|_{q} .
$$

Since the function

$$
f_{0}(x, y)=\frac{M}{\|K(\cdot, \cdot)\|_{q}^{q / p}} \int_{0}^{1} \int_{0}^{1}|K(t, u)|^{q-1} \operatorname{sgn} K(t, u) g_{1}(x, t) g_{2}(y, u) d t d u
$$

belongs to the set $W_{8_{1} g_{2}}^{2 r} L_{p}$, it follows from (8) that $E\left(f_{0}\right)=M\|K(\cdot, \cdot)\|_{q}$. Therefore we have from (9) $E=M\|K(\cdot, \cdot)\|_{q}$.

By the result on "polynomials" of least deviation from zero [5], we have that

$$
\begin{aligned}
& \quad \inf _{\left\{\alpha_{k}, \beta_{j}, \gamma_{k}, x_{k}, y_{j}\right\}}\|K(\cdot, \cdot)\|_{q} \\
& =\inf _{\left\{\alpha_{k}, \beta_{j}, x_{k}, y_{j}\right\}} \| \varphi_{1}(x) \varphi_{2}(y)-\varphi_{2}(y) \sum_{k=1}^{n} \alpha_{k} g_{1}\left(x_{k}, x\right) \\
& \quad-\varphi_{1}(x) \sum_{j=1}^{n} \beta_{j} g_{2}\left(y_{j}, y\right)+\sum_{k=1}^{n} \sum_{j=1}^{n} \alpha_{k} \beta_{j} g_{1}\left(x_{k}, x\right) g_{2}\left(y_{j}, y\right) \|_{q} \\
& =\inf _{\left\{\alpha_{k}, x_{k}\right\}}\left\|\varphi_{1}(\cdot)-\sum_{k=1}^{n} \alpha_{k} g_{1}\left(x_{k}, \cdot\right)\right\| \cdot \inf _{\left\{\beta_{j}, y_{j}\right\}}\left\|\varphi_{2}(\cdot)-\sum_{j=1}^{n} \beta_{j} g_{2}\left(y_{j}, \cdot\right)\right\|_{q} .
\end{aligned}
$$

As the equalities

$$
\begin{aligned}
& \inf _{\left\{\alpha_{k}, x_{k}\right\}}\left\|\varphi_{1}(\cdot)-\sum_{k=1}^{n} \alpha_{k} g_{1}\left(x_{k}, \cdot\right)\right\|_{q}=r_{p 1}^{(n)}, \\
& \inf _{\left\{\beta_{j}, y_{j}\right\}}\left\|\varphi_{2}(\cdot)-\sum_{j=1}^{n} \beta_{j} g_{2}\left(y_{j}, \cdot\right)\right\|_{q}=r_{p 2}^{(n)}
\end{aligned}
$$

hold and are achieved by the coefficients and knots (6) [4], it follows from (10) that the numbers (6) and (7) are the coefficients, knots, and estimate of the optimal formula (5) for the set $W_{g_{1} g_{2}}^{2 r} L_{p}$. 
The theorem is proved.

Now we will obtain a cubature formula which requires less computational work than the optimal formula (4) with the same error estimate.

Let

$$
\sup _{0 \leqslant y \leqslant 1}\left\|\frac{\partial^{r} f(\cdot, y)}{\partial x^{r}}\right\|_{p} \leqslant M_{1}, \quad \sup _{0 \leqslant x \leqslant 1}\left\|\frac{\partial^{r} f(x, \cdot)}{\partial y^{r}}\right\|_{p} \leqslant M_{2} .
$$

Then

$$
M_{1}^{-1} f\left(x, y_{p j}^{(n)}\right) \in W_{g_{1}}^{r} L_{p}, \quad M_{2}^{-1} f\left(x_{p k}^{(n)}, y\right) \in W_{g_{2}}^{r} L_{p} .
$$

Applying optimal quadrature formulas for these sets to the integrals in (5), we obtain

$$
\begin{aligned}
& \int_{0}^{1} f\left(x, y_{p j}^{(n)}\right) d x=\sum_{k=1}^{n^{2}} A_{p k}^{\left(n^{2}\right)} f\left(x_{p k}^{\left(n^{2}\right)}, y_{p j}^{(n)}\right)+r_{1}(f), \\
&\left|r_{1}(f)\right| \leqslant M_{1} r_{p 1}^{\left(n^{2}\right)} \\
& \int_{0}^{1} f\left(x_{p k}^{(n)}, y\right) d y=\sum_{j=1}^{n^{2}} B_{p j}^{\left(n^{2}\right)} f\left(x_{p k}^{(n)}, y_{p j}^{\left(n^{2}\right)}\right)+r_{2}(f), \\
&\left|r_{2}(f)\right| \leqslant M_{2} r_{p 2}^{\left(n^{2}\right)}
\end{aligned}
$$

Designating

$$
a_{n}=\left|A_{p 1}^{(n)}\right|+\cdots+\left|A_{p n}^{(n)}\right|, \quad b_{n}=\left|B_{p 1}^{(n)}\right|+\cdots+\left|B_{p n}^{(n)}\right|
$$

and substituting (11) and (12) into the optimal formula (5) for the set $W_{g_{1} g_{2}}^{2 r} L_{p}$, we obtain the following cubature formula

$$
\begin{aligned}
\int_{0}^{1} \int_{0}^{1} f(x, y) d x d y= & \sum_{k=1}^{n} \sum_{j=1}^{n^{2}} A_{p k}^{(n)} B_{p j}^{\left(n^{2}\right)} f\left(x_{p k}^{(n)}, y_{p j}^{\left(n^{2}\right)}\right) \\
& +\sum_{j=1}^{n} \sum_{k=1}^{n^{2}} B_{p j}^{(n)} A_{p k}^{\left(n^{2}\right)} f\left(x_{p k}^{\left(n^{2}\right)}, y_{p j}^{(n)}\right) \\
& -\sum_{k=1}^{n} \sum_{j=1}^{n} A_{p k}^{(n)} B_{p j}^{(n)} f\left(x_{p k}^{(n)}, y_{p j}^{(n)}\right)+E_{1}(f),
\end{aligned}
$$

where

$$
\left|E_{1}(f)\right| \leqslant a_{n} M_{2} r_{p 2}^{\left(n^{2}\right)}+b_{n} M_{1} r_{p 1}^{\left(n^{2}\right)}+M r_{p 1}^{(n)} r_{p 2}^{(n)}
$$

It follows from the convergence of optimal formulas (2) that $a_{n}$ and $b_{n}$ are bounded as $n \rightarrow \infty$. Hence it follows from (14) that

$$
\left|E_{1}(f)\right|=O\left(n^{-2 r}\right) \text {. }
$$

The formula (13) has a remarkable advantage over the optimal formula (4). The optimal formula (4) with $m=n^{2}$ has the error estimate $O\left(n^{-2 r}\right)$, and it uses $n^{4}$ point values of $f(x, y)$, while the formula (13) has the same error estimate $O\left(n^{-2 r}\right)$ but uses only $2 n^{3}+n^{2}$ point values of the function $f(x, y)$. 
3. Example. We compare the evaluation of the integral

$$
I=\int_{0}^{1} \int_{0}^{1} \frac{\left(x-x^{2}\right)\left(y-y^{2}\right)}{0 \cdot 2+x y} d x d y=0.0701598 \ldots
$$

with the optimal formula (4) with $m=n^{2}$ and with the formula (13) obtained from the optimal formula (5). Both formulas are taken for the set $W_{g_{1} g_{2}}^{2 r} L_{2}, r=2$.

As the integrand $f(x, y)$ satisfies the conditions

$$
f(0, y) \equiv f(1, y) \equiv f(x, 0) \equiv f(x, 1) \equiv 0,
$$

we can take the functions $g_{1}(x, t) \equiv g_{2}(x, t)$ as Green's functions for the problem

$$
y^{\prime \prime}=0, \quad y(0)=y(1)=0 \text {. }
$$

Then by [4] we have

$$
\begin{gathered}
A_{2 k}^{(m)}=B_{2 k}^{(m)}=2 \varepsilon, \quad k=2, \ldots, m-1, \\
A_{21}^{(m)}=B_{21}^{(m)}=A_{2 m}^{(m)}=B_{2 m}^{(m)}=(1+1.25 \sqrt{2 / 3}) \varepsilon, \\
x_{2 k}^{(m)}=y_{2 k}^{(m)}=2 \varepsilon(\sqrt{2 / 3}+k-1), \quad k=1, \ldots, m, \\
\varepsilon=0.5(2 \sqrt{2 / 3}+m-1)^{-1} .
\end{gathered}
$$

Let $I_{n}$ be an approximate value of $I$, obtained by the optimal formula (4) with $k_{n}=n^{4}$ knots and let $I_{n}^{\prime}$ be the approximate value obtained by formula (13) with $q_{n}=2 n^{3}+n^{2}$ knots.

We obtain for $n=4,7,9$

\begin{tabular}{ccccc}
\hline$I_{n}^{\prime}$ & $I_{n}$ & $q_{n}$ & $k_{n}$ & $n$ \\
\hline 0.0701302 & 0.0701319 & 144 & 256 & 4 \\
0.0701587 & 0.0701588 & 735 & 2401 & 7 \\
0.0701596 & 0.0701596 & 1539 & 6561 & 9 \\
\hline
\end{tabular}

The superiority of formula (13) over the optimal formula (4) is obvious.

Acknowledgement. I am grateful to Professor Nira Dyn for stimulating discussions on the material presented in this paper.

School of Mathematical Sciences

Tel-Aviv University

Ramat-Aviv, Tel-Aviv, Israel

1. F. J. Delvos \& H. Posporf, “N-th order blending," in Constructive Theory of Functions of Several Variables (W. Schempp and K. Zeller, eds.), Springer-Verlag, Berlin, Heidelberg, New York, 1977, pp. 53-64.

2. W. J. GORDON, "Distributive lattices and the approximation of multivariate functions," in $A p$ proximations with Special Emphasis of Spline Functions (I. J. Schoenberg, ed.), Academic Press, New York, London, 1969, pp. 223-277.

3. M. Levin, "On approximation in $L_{2}$ and optimal cubature formulae," in Fourier Analysis and Approximation Theory, Mathematica Societatis János Bolyai, Budapest, 1976, pp. 495-501.

4. M. Levin \& J. Girshovich, Optimal Quadrature Formulas, Teubner-Verlag, Leipzig, 1979.

5. M. Levin \& J. GirShovich, "Extremal problems for cubature formulas," Soviet Math. Dokl., v. 18, 1977, pp. $1355-1358$. 